\title{
High Frame Rate Ultrasound Particle Image Velocimetry for Estimating High Velocity Flow Patterns in the Left Ventricle
}

\author{
Jason Voorneveld ${ }^{\mathbb{\infty}}$, Graduate Student Member, IEEE, Aswin Muralidharan ${ }^{\mathbb{(}}$, Timothy Hope, \\ Hendrik J. Vos, Member, IEEE, Pieter Kruizinga, Member, IEEE, \\ Antonius F. W. van der Steen, Fellow, IEEE, Frank J. H. Gijsen, \\ Sasa Kenjeres, Nico de Jong, Member, IEEE, \\ and Johan G. Bosch, Member, IEEE
}

\begin{abstract}
Echocardiographic determination of multicomponent blood flow dynamics in the left ventricle remains a challenge. In this paper, we compare contrast enhanced, high frame rate (HFR) (1000 frames/s) echo-particle image velocimetry (ePIV) against optical particle image velocimetry (oPIV, gold standard), in a realistic left ventricular $(\mathrm{LV})$ phantom. We find that ePIV compares well to oPIV, even for the high velocity inflow jet (normalized $\mathrm{RMSE}=9 \% \pm 1 \%$ ). In addition, we perform the method of proper orthogonal decomposition, to better qualify and quantify the differences between the two modalities. We show that ePIV and oPIV resolve very similar flow structures, especially for the lowest order mode with a cosine similarity index of $86 \%$. The coarser resolution of ePIV does result in increased variance and blurring of smaller flow structures when compared to oPIV. However, both modalities are in good agreement with each other for the modes that constitute the bulk of the kinetic energy. We conclude that HFR ePIV can accurately estimate the high velocity diastolic inflow jet and the high energy flow structures in an $\mathrm{LV}$ setting.
\end{abstract}

Index Terms-Echo-particle image velocimetry (ePIV), echocardiography, high frame rate (HFR) imaging, left ventricular (LV) flow, ultrasound contrast agents (UCAs), ultrasound imaging velocimetry (UIV).

\section{INTRODUCTION}

$\mathbf{T}$ HE left ventricular (LV) diastolic filling vortex has been suggested as a potential early-stage biomarker for cardiac dysfunction [1]-[7]. At present, Doppler echocardiography and phase-contrast cardiovascular magnetic resonance (PC-CMR) imaging are the primary techniques used for clinical assessment of LV filling dynamics. However, Doppler echocardiography is limited to assessing a single velocity component along the beam axis; and whereas PC-CMR can resolve

Manuscript received August 31, 2017; accepted December 18, 2017. Date of publication December 22, 2017; date of current version December 20, 2018. This work was supported by ZonMw within the Innovative Medical Devices Initiative Program under Project Heart Failure and 4-D Flow. (Corresponding author: Jason Voorneveld.)

J. Voorneveld, T. Hope, H. J. Vos, P. Kruizinga, A. F. W. van der Steen, F. J. H. Gijsen, N. de Jong, and J. G. Bosch are with the Department of Biomedical Engineering, Thoraxcenter, Erasmus Medical Center, 3015CN Rotterdam, The Netherlands (e-mail: j.voorneveld@erasmusmc.nl).

A. Muralidharan and S. Kenjeres are with the Department of Chemical Engineering, Transport Phenomena Section, Faculty of Applied Sciences, Delft University of Technology, 2629 HZ Delft, The Netherlands.

Digital Object Identifier 10.1109/TUFFC.2017.2786340 velocity components in 3-D, its temporal resolution is limited, and acquisition is phase averaged over hundreds of cardiac cycles, resulting in the blurring of cycle-to-cycle variation, which may contain information of clinical importance [8].

Several ultrasonic techniques have emerged to measure blood flow in 2-D or 3-D rather than along the beam direction. Vector Doppler imaging (VDI, also known as multiangle Doppler) is one such technique developed to measure both the axial- and cross-beam flow component; this is achieved by retrieving at least two Doppler measurements at a known angle to each other, which can then be used to deduce the separate flow components [9]. However, VDI requires a large aperture for imaging deep structures, which is impractical in transthoracic echocardiography due to the small intercostal windows.

Transverse oscillation (TO) is a similar technique to VDI, in that it splits the transducer aperture, but in this case it does so synthetically by applying an apodization function in receive. TO has been used to measure LV blood flow but is limited to open-chest scanning only due to depth limitations [10], [11]. A recent variation of TO, named directional TO (DTO), is a method to automatically calibrate the TO technique with depth. DTO has shown reasonable accuracy at depths of up to $160 \mathrm{~mm}$ in pipe flow experiments, although its use in complex flow environments has yet to be demonstrated [12].

Alternatively, in a more numerical approach, vector flow mapping combines conventional 2-D color Doppler with tracking of the LV wall motion, and applies a mass-conservation constraint (under the assumption of planar flow) to numerically estimate the cross-beam velocity component at each point in the velocity field [13]. A similar technique, referred to as echo dynamography, estimates the cross-beam components utilizing only color-Doppler measurements. It achieves this by splitting the flow field (assumed to be laminar) into a nonvortical and several vortical flow components; of which the axial- and cross-beam velocity components can be derived [14].

Along an image-processing-based paradigm, blood speckle tracking estimates the displacement of local distributions of red blood cells (RBC) [15]. This technique has been used for flow estimation in neonates [16] and pediatric patients [17]. However, for adult cardiac applications, this technique requires 


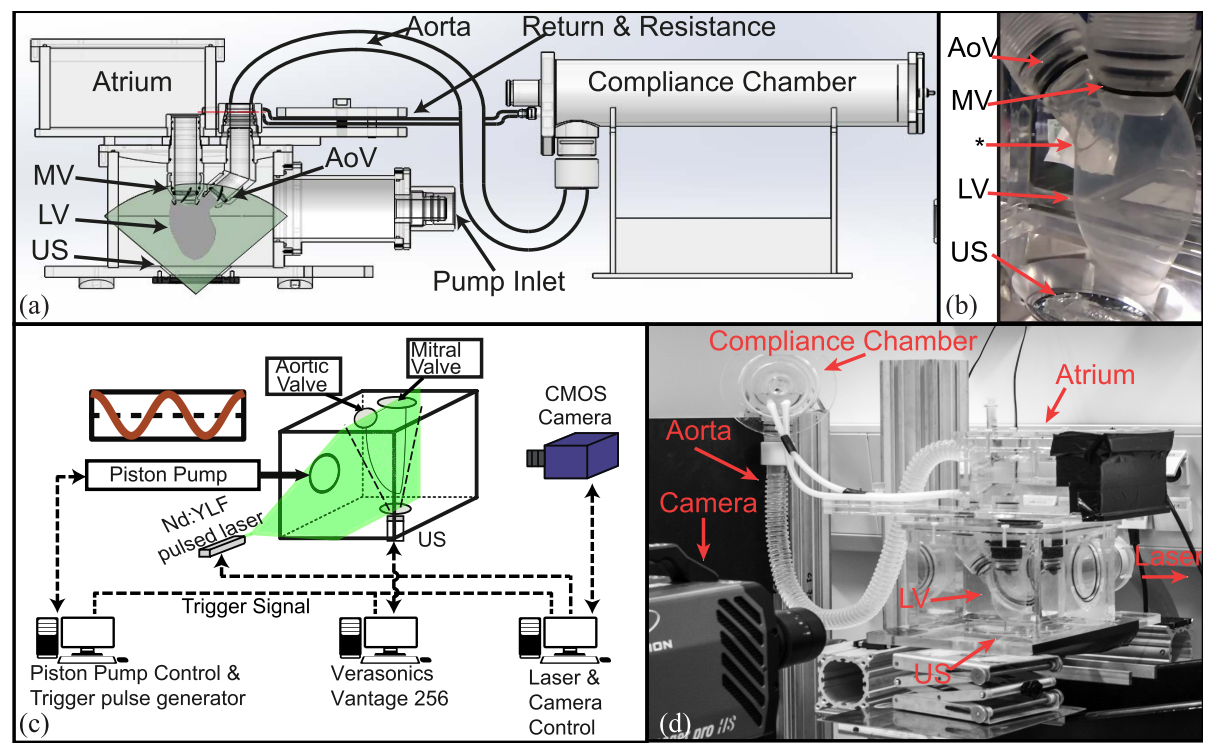

Fig. 1. LV phantom and experimental setup. (a) Diagrammatic representation of LV phantom. (b) Zoomed-in view of the compliant LV with aortic (AoV) and mitral (MV) valves and the US transducer through its view port. * Note the opaque joint region at the start of the aortic outflow tract. (c) Diagram of experiment: control of laser, US, and pump are all performed with different computers and synchronization is achieved by a trigger pulse provided by the pump control system. (d) Photograph of ePIV versus oPIV experimental setup.

a high signal-to-noise ratio (SNR) to retrieve sufficient signal from the blood, after clutter filtration. Here ultrasound contrast agent (UCA) can be beneficial, providing a large SNR improvement over the scattered signals from RBC. UCA has widely been used in a similar technique to blood speckle tracking named echo-particle image velocimetry (ePIV, also known as ultrasound imaging velocimetry or UIV).

ePIV estimates the local displacement of a sparse distribution of microbubbles. However, when using focused transmission schemes, ePIV accuracy diminishes in the presence of fast flow. This is due to the relatively large scatterer displacement, and the associated speckle decorrelation, between frames [18]. The inability to resolve fast flows is especially detrimental to investigating the effect of the diastolic filling vortex, where the velocities inside the jet can exceed $1 \mathrm{~m} / \mathrm{s}$. Indeed, previous LV flow phantom studies utilizing ePIV have both mentioned the limitations of the method with regard to tracking the high velocity transmitral jet [19], [20]. However, the recent feasibility of high frame rate (HFR) US acquisitions, using unfocussed transmit protocols, has offered a possible solution to the dynamic range limitations of ePIV.

Indeed, our previously reported work [21] and a study by Leow et al. [22] verified that the ultrafast ePIV could accurately estimate high velocity flows in a blood vessel setting. However, these experiments do not cover the transient and multidimensional nature of intraventricular flow. Expanding on our previous work, we have developed an acoustically and optically transparent LV flow phantom to be used for optical PIV and US.

In this paper, we investigate whether HFR ePIV can accurately measure high velocity intraventricular flows. To do so, we simultaneously acquire HFR US and time resolved digital PIV (herein referred to as optical PIV, i.e., oPIV) acquisitions at equivalent frame rates, allowing for frame-toframe comparison between the two modalities.

\section{Methods}

\section{A. Left Ventricular Phantom}

A compliant, optically and acoustically transparent silicone LV chamber [Fig. 1(b)], was manufactured by painting four layers of silicone (HT 33 Transparente LT, Zhermack SpA, Rome, Italy) onto a 3-D-printed mold of an LV. The 3-D-printed mold was modeled from the statistical mean (end systolic phase) of a data set of segmented 4-D computed tomography images of 150 patients [23], [24]. The silicone LV was then fit with mitral [Fig. 1(b)-MV] and aortic [Fig. 1(b)-AoV] Björk-Shiley valves.

The LV was encased in a transparent acrylic box and fit with mitral and aortic valve ports, which were connected to an atrial chamber and an aortic compliance chamber, respectively (Fig. 1). The acrylic box had one open port, which was connected to a programmable piston pump, which reciprocated in a sinusoidal pattern at a frequency of $1 \mathrm{~Hz}$ with an $80-\mathrm{ml}$ stroke volume. The pump control system additionally provided a trigger pulse for synchronized acquisition between the oPIV and ePIV systems. The LV phantom design was inspired by Gao et al. [19]; however, this phantom is also designed to be MRI compatible for future research.

The LV housing had three flat and transparent surfaces for laser/camera view access, as well as an US port on the underside of the box, sealed with a thin film. The orientation of the US and laser/camera ports allow for simultaneous acquisition of oPIV and ePIV recordings.

The system works with two incompressible fluid systems: 1) the hydraulic fluid system, which is contained in the LV enclosure and transfers energy from the pump to the LV and 2) the "blood" fluid system, which flows from the atrium through the LV to the compliance chamber, slowly refilling through the return lines. The two fluid systems do not mix and the "blood" fluid system dynamically pumps in response 
to the compression and expansion of the silicone LV wall, which follows the volume change in the hydraulic fluid system induced by the piston pump.

Both fluids were $66 \%$ glycerol in water solutions (density $=$ $1160 \mathrm{~kg} / \mathrm{m}^{3}$ and viscosity $=0.0177 \mathrm{~Pa} \cdot \mathrm{s}$ ) to allow for correct optical index matching between the silicone of the LV and the fluid. The mismatch in density and viscosity to normal LV blood values means that the Reynolds number is not matched to in vivo values. However, in this paper, we are primarily interested in capturing the diastolic, transmitral jet at similar velocities to those observed in vivo $(\sim 1 \mathrm{~m} / \mathrm{s})$.

The pump control system was not optimal, in that the pump stroke between acquisitions was not consistent. However, as oPIV and ePIV were acquired simultaneously this did not adversely affect the comparison.

\section{B. Echo-Particle Image Velocimetry}

1) Ultrasound Settings: US RF data were acquired with a Verasonics Vantage 256 system (Verasonics Inc., Kirkland, WA, USA) using a curvilinear (3 MHz, C5-2, and ATL) probe. A single pulselength diverging wave, three-angled acquisition protocol was used at a pulse repetition frequency of $3 \mathrm{kHz}$, resulting in imaging at $1 \mathrm{kHz}$ after correlation compounding (see Section II-2). Data sets were acquired for 2 s, starting when the trigger pulse from the pump controller was received, allowing for two full heart cycles to be captured.

Before beamforming, a $70 \mathrm{~Hz}$, eighth-order, high-pass Butterworth filter was applied to the channel data in the slow-time dimension to remove static clutter. The Verasonics Vantage software (V.3.0.7, all postprocessing turned OFF) was used for IQ reconstruction, and the envelope data were used as an input for the ePIV algorithm.

An UCA(UCA, SonoVue, Bracco S.p.A) was used as a tracer particle for ePIV tracking. The UCA was injected in a $0.25-\mathrm{ml}$ bolus injection, resulting in a concentration of approximately $90 \mu \mathrm{l} / 1$.

2) ePIV Settings: We used a modified version of PIVlab [25] as an ePIV implementation in MATLAB (R2017a, The MathWorks Inc., Natick, USA). This modified implementation performed block-wise cross correlation for each corresponding angle (in this case three angles), averaging their correlation maps before peak finding (Fig. 2). This was performed to reduce the decorrelation effects of bubble motion between angles.

An iterative scheme is employed where the interrogation window is resized and deformed using the displacement estimates from the previous iteration before performing the next iteration of displacement estimation. Deformation was performed using linear interpolation. Four iterations were performed in total with the first two having a kernel size of $64 \times 64$ pixels $(\sim 18 \times 18 \mathrm{~mm})$ and the last two refined to $32 \times 32$ pixels $(\sim 9 \times 9 \mathrm{~mm})$, all with an overlap of $75 \%$. Pixel resolution was $280 \mu \mathrm{m}(\lambda / 2)$ and the final grid spacing was $2.3 \mathrm{~mm} \times 2.3 \mathrm{~mm}$. Cross correlation was performed in the Fourier domain and to reduce the implicit bias toward zero displacement (caused by loss of particle pairs) the correlation maps were corrected by an appropriate window function [26].

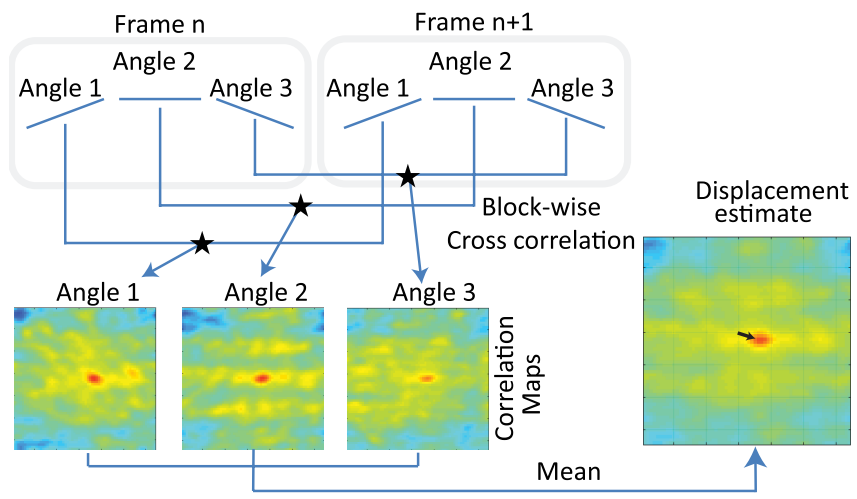

Fig. 2. Illustration of the angle-wise cross correlation averaging technique used for ePIV estimation. Corresponding angles between successive image frames are used for cross correlation comparison and the mean correlation map of all angles is used for peak finding. This process is repeated multiple times per frame pair after interrogation window refinement and deformation.

For post-processing, both local median test [27] $(\varepsilon=0.05$, threshold $=3$, and $b=1$ ) and global standard deviation $(\sigma=3)$ outlier tests were performed, replacing removed vectors with interpolated values from surrounding data. Finally, a moving average filter (five ensembles) was applied along the time dimension of the data and a Gaussian convolution filter $(\sigma=0.5 \times 0.5$ and span $=3 \times 3$ ) was used spatially. Table I provides a summary of the implementation details and parameters used for ePIV.

\section{Optical Particle Image Velocimetry}

The oPIV was used to measure the instantaneous 2-D velocity field in sync with ePIV, which was performed at the same frame rate. The oPIV measurement plane was aligned to the US plane and was positioned such that both the AoV and MV would be visible in the plane, allowing for both filling and ejection dynamics to be captured. The oPIV setup is photographed in Fig. 1(d) and summarized in Fig. 1(c) and Table I. Briefly, the oPIV setup consisted of a high-speed CMOS camera (Imager Pro HS 4M, LaVision Inc., Bicester, U.K.) equipped with a 100-mm focal length lens arranged perpendicular to the measurement plane. Image calibration was performed using a two-level calibration plate (106-10, LaVision Inc.) placed in the desired measurement plane.

The camera's field of view was adjusted to narrowly include the entire ventricle in an area of approximately $95 \times 95 \mathrm{~mm}^{2}$ at a focal aperture of 5.6. The measurement plane was illuminated by a high-speed dual cavity pulsed Nd:YLF laser (526 nm, LDY304, Litron Lasers, Rugby, U.K.). The laser sheet thickness was approximately $1 \mathrm{~mm}$ and the camera depth of field was approximately $1.8 \mathrm{~mm}$. The 2000 frames were acquired over a 2-s interval following the trigger signal received from the pump control system, thus synchronizing the ePIV and oPIV acquisitions.

The hollow glass spheres used as oPIV tracers were not visible in the US images, and the microbubbles used for ePIV were not visible in the oPIV images. 
TABLE I

PIV PARAMETERS

\begin{tabular}{|c|c|c|c|}
\hline \multirow{7}{*}{$\frac{\text { Parameter }}{\text { Transmit }}$} & \multirow{7}{*}{$\begin{array}{l}\text { oPIV } \\
\text { 150W, 527-nm pulsed } \\
\text { Nd:YLF Laser } \\
\text { Pulse interval: } 1 \mathrm{~ms} \\
\text { Pulse duration: } 150 \mathrm{~ns}\end{array}$} & \multicolumn{2}{|l|}{ ePIV } \\
\hline & & Transducer: & C5-2 (ATL) \\
\hline & & \multicolumn{2}{|c|}{ Center frequency: $3.1 \mathrm{MHz}$} \\
\hline & & \multicolumn{2}{|c|}{ Pulse interval: $\quad 0.33 \mathrm{~ms}$} \\
\hline & & \multirow{2}{*}{$\begin{array}{l}\text { Pulse duration: } \\
\text { No. of angles: }\end{array}$} & 1 cycle \\
\hline & & & $\begin{array}{l}3\left(-10.5^{\circ}, 0^{\circ},\right. \\
\left.10.5^{\circ}\right)\end{array}$ \\
\hline & & \multicolumn{2}{|l|}{ Apodization: } \\
\hline \multirow[t]{8}{*}{ Receive } & $\begin{array}{l}2016 \times 2016 \text { pixel, } \\
12 \text { bit CMOS Camera }\end{array}$ & US Scanner: & $\begin{array}{l}\text { Verasonics } \\
\text { Vantage } 256\end{array}$ \\
\hline & Sensor size: $11 \mu \mathrm{m}$ & No. of elements: & 128 \\
\hline & te: $1 \mathrm{kHz}$ & Acquisition rate: & $3 \mathrm{kHz}$ \\
\hline & Viewing angle: $90^{\circ}$ & Opening angle: & $75^{\circ}$ \\
\hline & Lens focal length: $100 \mathrm{~mm}$ & Focal depth: & $60 \mathrm{~mm}$ \\
\hline & Lens focal aperture: 5.6 & & \\
\hline & FOV: $\approx 95 \times 95 \mathrm{n}$ & FOV: & $110 \mathrm{~mm} \times 75^{\circ}$ \\
\hline & & Apodization: & Tukey $(\alpha=0.2)$ \\
\hline$\overline{\text { Particles }}$ & $\begin{array}{l}8-12 \mu \mathrm{m} \text { hollow glass } \\
\text { spheres }\end{array}$ & \multicolumn{2}{|c|}{$\begin{array}{l}1-10 \mu \mathrm{m} \text { micro-bubbles } \\
(\text { SonoVue }(), 0.25 \mathrm{ml} \text { bolus }\end{array}$} \\
\hline \multirow[t]{3}{*}{$\begin{array}{l}\text { Vector } \\
\text { Analysis }\end{array}$} & $\begin{array}{l}\text { Multi-pass, normalized } \\
\text { FFT cross correlation with } \\
\text { window refinement: } \\
64 \times 64 \text { ( } 2 \text { pass) to } 16 \times 16 \\
(5 \text { pass })\end{array}$ & \multicolumn{2}{|c|}{$\begin{array}{l}\text { Multi-angle, multi-pass, } \\
\text { normalized FFT cross correlation } \\
\text { with window refinement: } \\
64 \times 64 \text { ( } 2 \text { pass) to } 32 \times 32 \text { ( } 2 \text { pass) }\end{array}$} \\
\hline & $50 \%$ overlap & \multicolumn{2}{|l|}{$75 \%$ overlap } \\
\hline & $\begin{array}{l}\text { Final grid spacing: } 375 \mu \mathrm{m} \\
\text { x } 375 \mu \mathrm{m}\end{array}$ & \multicolumn{2}{|c|}{$\begin{array}{l}\text { Final grid spacing: } 2.3 \mathrm{~mm} \times 2.3 \\
\mathrm{~mm}\end{array}$} \\
\hline \multirow{3}{*}{$\begin{array}{l}\text { Validation } \\
\text { and } \\
\text { Smoothing }\end{array}$} & Universal outlier detection & \multirow{3}{*}{\multicolumn{2}{|c|}{$\begin{array}{l}\text { Universal outlier detection } \\
\text { Global standard deviation filter } \\
3 \times 3 \text { Gaussian spatial filter } \\
5 \text { ensemble moving average }\end{array}$}} \\
\hline & Single pass de-noising & & \\
\hline & $\begin{array}{l}(3 \times 3) \\
3 \text { ensemble moving average }\end{array}$ & & \\
\hline
\end{tabular}

Vector calculation was performed using the TRDPIV package in the Davis 8.4 (LaVision Inc., U.K.) software package. This implementation used an iterative fast Fourier transform cross-correlation scheme with interrogation window refinement, ranging from $64 \times 64$ pixels down to $16 \times 16$ pixels with 50\% overlap throughout. Pixel resolution was $47 \mu \mathrm{m}$ and final grid spacing was $375 \mu \mathrm{m} \times 375 \mu \mathrm{m}$.

\section{Comparison of oPIV and ePIV}

The velocity profile of the primary inflow jet was used to compare the two modalities. In addition, a technique widely used for flow feature studies in oPIV and numerical analysis called proper orthogonal decomposition (POD) was used for comparison of the dominant flow features observed through oPIV and ePIV [28]-[30]. Using a conventional vector-tovector analysis would require precise alignment between the US and laser scanning planes which is impractical. Using POD, the dominant flow structures observed in the two data sets could reliably be compared.

Before comparison, the oPIV data set was spatially down sampled (using a local mean) to match the grid spacing of the ePIV data. This made for a more meaningful comparison to the ePIV data which is an order of magnitude lower in resolution than oPIV. It was also required for POD analysis, in order to match the energy density between the two data sets.

\section{E. Proper Orthogonal Decomposition (POD)}

The POD technique (described in detail in [29] and [30]) has been extensively used and developed in the fluid dynamics community but a brief description will be provided here.
POD analysis decomposes a time-sampled vector field into a set of orthogonal basis vectors (modes), and a set of scalar weighting coefficients (2). The utility of the method lies in that the modes and their corresponding coefficients are ordered in a descending manner by their energy contribution to the observed data set. Thus, large (spatially and in magnitude) and coherent flow structures are described in the low-order modes; whereas small and incoherent flow structures are shifted toward the high-order modes. A typical POD results in the majority of energy being contained in a minority of the low-order modes. Hence, a comparison of the first few modes between decomposed data sets can reveal similarity or differences between the two data sets, largely reducing the dimensionality of the comparison problem [28], [32].

In this paper, the method of snapshots was used [33] which provides a discrete approximation of the POD method in the temporal domain. First, the velocity grids are reorganized into a concatenated Casorati matrix $\boldsymbol{U}$

$$
U=\left[u^{1} u^{2} \ldots u^{N}\right]=\left[\begin{array}{cccc}
u_{x 1}^{1} & u_{x 1}^{2} & \ldots & u_{x M}^{N} \\
\vdots & \vdots & \ddots & \vdots \\
u_{x M}^{1} & u_{x M}^{2} & \ldots & u_{x 1}^{N} \\
u_{y 1}^{1} & u_{y 1}^{2} & \ldots & u_{y 1}^{N} \\
\vdots & \vdots & \ddots & \vdots \\
u_{y M}^{1} & u_{y M}^{2} & \ldots & u_{y M}^{N}
\end{array}\right]
$$

where $u_{x}$ and $u_{y}$ are the velocity components in the axial and azimuth directions, respectively, $\mathbf{N}$ is the number of frames in the sequence, and $\mathbf{M}$ is the number of vectors in the field per frame.

POD decomposes $\mathbf{U}$ into a set of orthogonal spatial modes $\Phi$ and a set of temporal weighting coefficients $\mathbf{A}$

$$
\boldsymbol{U}=\boldsymbol{\Phi} \boldsymbol{A} .
$$

To calculate $\Phi$ and $\mathbf{A}$, the covariance matrix $\mathbf{C}$ is computed

$$
C=U^{T} U
$$

from which the eigenvalue equation can be solved

$$
C \beta=\lambda \beta
$$

where the eigenvalues, $\lambda$, are ordered in decreasing amplitude.

The eigenvectors $\beta$ are used to construct the POD modes $\varphi^{i}$

$$
\varphi^{i}=\frac{\sum_{n=1}^{N} \beta_{n}^{i} u^{n}}{\lambda^{i}}, \quad i=1, \ldots, N .
$$

The POD coefficients are calculated by projecting the velocity components onto the POD modes

$$
a^{n}=\Phi^{T} u^{n}
$$

where $\boldsymbol{\Phi}=\left[\boldsymbol{\varphi}^{1} \boldsymbol{\varphi}^{2} \ldots \boldsymbol{\varphi}^{n}\right]$ and $\boldsymbol{A}=\left[\boldsymbol{a}^{1} \boldsymbol{a}^{2} \ldots \boldsymbol{a}^{n}\right]^{T}$.

To ensure that modes between the two data sets were comparable (i.e., shared), a combined decomposition was performed. That is, the vectors of each data set were concatenated before POD and then reconstituted afterward for comparison. 

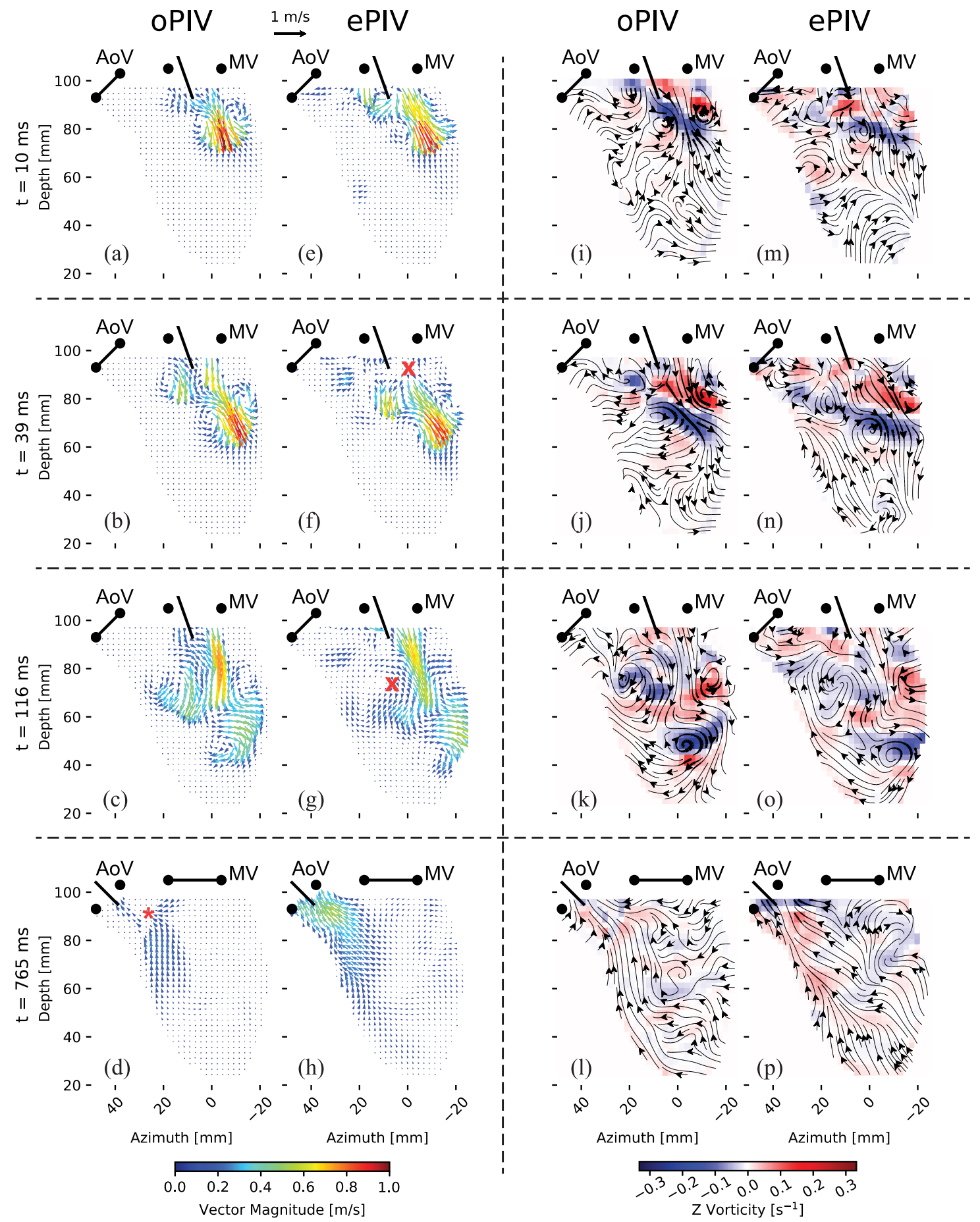

Fig. 3. Vector field comparison between (a)-(d) oPIV and (e)-(h) ePIV during (a)-(c) and (e)-(g) filling and (d) and (h) ejection. Vorticity and streamline comparison between (i)-(1) oPIV and (m)-(p) ePIV. ePIV is able to capture the high velocity inflow jet, and similar flow patterns are observed. (d) * Note that oPIV cannot resolve vectors in the vicinity of the opaque connection to the outflow tract [see Fig. $1(\mathrm{~d})$ — $^{*}$ ]. (g) $\mathbf{X}$ : ePIV tracking errors due to transient clutter artifact (see text). Black lines and dots represent the disks and seats, respectively (indicating a closed position when they overlap). A video showing one full cycle is available at http://ieeexplore.iee.org $\mathrm{E}$. $\mathrm{foV}=$ aortic valve and $\mathrm{MV}=$ mitral valve.

Similarity between the POD modes of oPIV and ePIV was quantitatively assessed using the cosine of the angle between their vectors where (,) the two sets of basis functions and $\|\cdot\|$ denotes the $L_{2}$ norm.

\section{RESULTS}

\section{A. Description of Flow}

Three frames during filling and a frame during ejection are shown in Fig. 3, where the first two columns (a)-(h) depict 
the velocity vector fields and the last two columns (i)-(p) depict the calculated vorticity fields with overlaid streamlines. In Fig. 3(a), (e), (i), and (m), the LV is imaged shortly after MV opening. Due to the geometry of the Björk-Shiley MV two jets develop on the lateral (right) and septal (left) sides of the MV [Fig. 3(b) and (f)]. The primary (lateral) jet has a higher velocity than the secondary (septal) jet, and accompanying contra-rotating vortices, visible in both the oPIV and ePIV measurements. This jet develops further with the lateral (right) vortex moving slightly apically but mostly remaining in the basal region (Fig. 3-second and third rows). Concurrently, the septal (left) vortex migrates apically before dissipating and splitting into smaller vortices. The primary jet develops into a continuous stream over time while simultaneously reducing in velocity magnitude. The secondary jet only forms a clockwise vortex [Fig. 3(k) and (o)] on the septal (left) side of the ventricle and dissipates much faster than the primary jet. During ejection (Fig. 3-last row), fluid is pushed toward the aortic outflow tract and fluid near the septal wall is observed to move with a higher velocity than in the center of the LV.

\section{B. Qualitative Comparison}

The similarity between ePIV and oPIV is visible in all phases of Fig. 3, with the exception of the ejection period where oPIV was not able to detect the velocities in the outflow tract due to the opaque joint in the silicone [Fig. 3(d) -*, caused by the manufacturing procedure of the LV phantom, see Fig. 1(b) - ${ }^{*}$ ]. However, the velocities in the aortic outflow tract region were resolvable by ePIV [Fig. 3(h)].

Two tracking errors of ePIV are observed in Fig. 3(f) and $(\mathrm{g})-\mathbf{X}$. Transient clutter caused by strong sidelobes of stationary air bubbles (on the LV wall) obscures the marked regions during the instances shown. The ePIV derived velocity vectors showed more frame-to-frame fluctuation than those derived from oPIV, especially in the basal region, which was in the elevational far-field of the US image (>80-mm depth).

\section{Quantitative Comparison}

The ability of ePIV to capture the full range of velocities present in the field is demonstrated in Fig. 4, where time profiles (from three separate acquisitions) through the jet cross section are plotted for both ePIV and oPIV. Note the high level of agreement between the two temporal profiles regardless of the variation in pump-induced flow profile. Indeed, the RMSE for the three repeated profiles in Fig. 4 was $5.5 \pm 0.1 \mathrm{~cm} / \mathrm{s}$ $(9 \% \pm 1 \%)$. However, ePIV still slightly underestimates the flow when compared to the oPIV, which is reflected in an RMSE of $10 \pm 1 \mathrm{~cm} / \mathrm{s}(16 \% \pm 2 \%)$, when only the high velocity $\left(\left|\mathbf{v}_{\text {mean }}\right|>30 \mathrm{~cm} / \mathrm{s}\right)$ portions of the cycle are taken into account.

\section{POD Qualitative Comparison}

To assess the similarity of the flow features observed by both modalities, a POD analysis was performed. The results of this analysis are shown in Figs. 5-7. Vector plots of the five
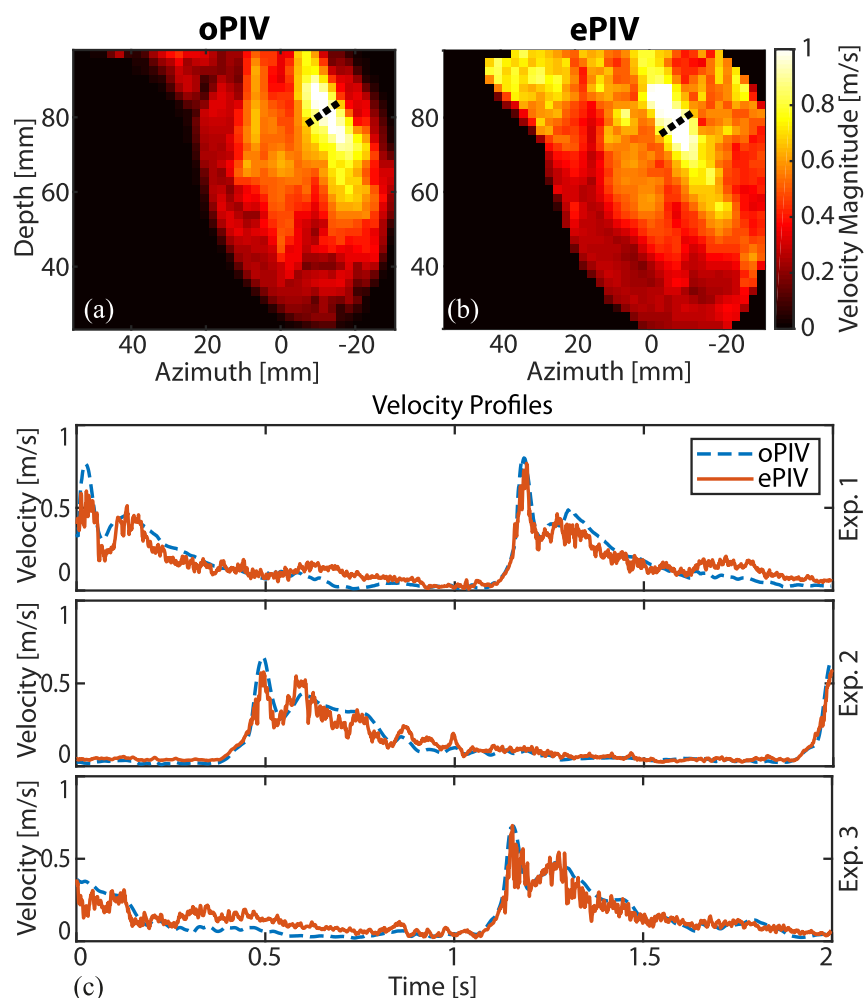

(c)

Fig. 4. Maximum intensity projections of the (a) oPIV and (b) ePIV time series. (c) Mean temporal velocity profiles in the jet region (of three repeated experiments), denoted by the dotted lines in (a) and (b), for oPIV (blue dashed) and ePIV (orange solid). Note that the good agreement between oPIV and ePIV even though the pump-induced flow profile was inconsistent between repetitions.

lowest order modes for ePIV and oPIV are shown in Fig. 5 (one row per mode). The POD coefficient profiles, in the azimuthal and axial directions, of the five lowest order modes are shown in Fig. 6. The minor underestimation of ePIV is apparent in the POD analysis, which can be seen in the axial coefficients of mode 1 (Fig. 6-region A), which for the most part, describes the primary jet stream [Fig. 5(a) and (f)]. Note that the secondary jet stream is not as prominent for ePIV as it is for oPIV in mode 1 [Fig. 5(a) and (f)]. During ejection, oPIV is not able to resolve flowthrough the opaque joint region (see Fig. 1(b) - $\left.{ }^{*}\right)$, whereas ePIV can; which is reflected in both their POD modes [Fig. 5(c) and (h)] and their coefficients (Fig. 6-*).

It should be noted that the flow profiles generated by the different modes are not necessarily real flow patterns that can be observed in the original data sets, they are instead the principal components of variation throughout the data set, where the low-order modes are of interest due to their inherent high kinetic energy. In the combined POD analysis, the first 53 modes (2.65\% of the total number of modes) comprised $95 \%$ of the total energy in the decomposition.

\section{E. POD Quantitative Comparison}

Quantitative comparison of the two modalities can be seen in Fig. 7(a), where the similarity index is plotted per mode 


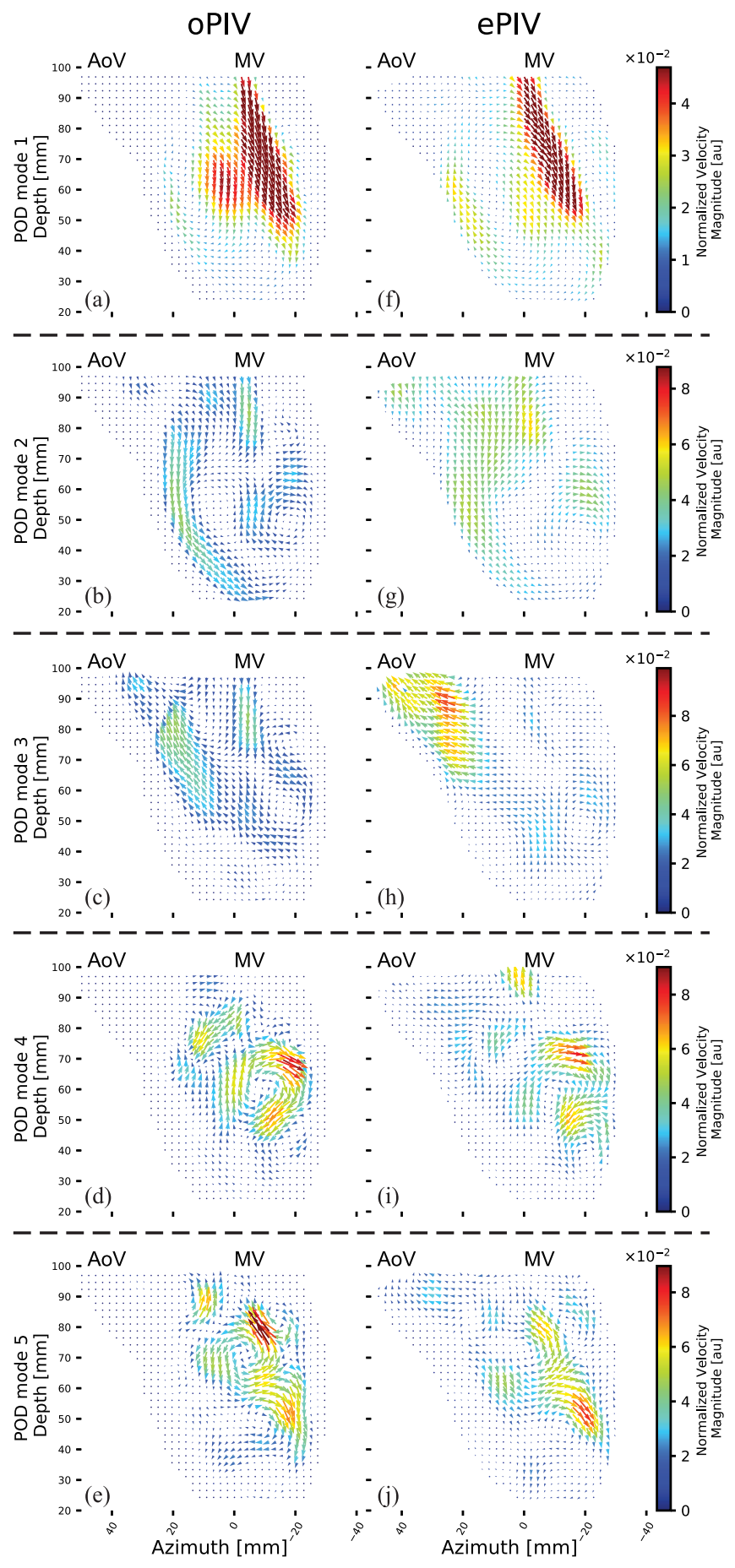

Fig. 5. Vector map plots of the first five POD modes for (a)-(e) oPIV and (f)-(j) ePIV. Good agreement is noted for all modes. Mode 1: the primary jet is very similar, but the secondary jet is weaker in ePIV. Mode 2: vector magnitude is more disperse for ePIV than oPIV. Mode 3: ejection dynamics captured by ePIV but not oPIV, similar to that observed in Fig. 3(d) and (h). Modes 4 and 5: POD starting to describe high-energy vortical structures, similarly captured for both modalities. AoV $=$ aortic valve and $\mathrm{MV}=$ mitral valve.

along with the cumulative energy fraction (CEF). The similarity is highest for the first few modes of variation but drops off for higher order modes. The first five modes have a similarity index ( $\mathrm{Rp}$ ) of above 0.5. These first five modes comprise

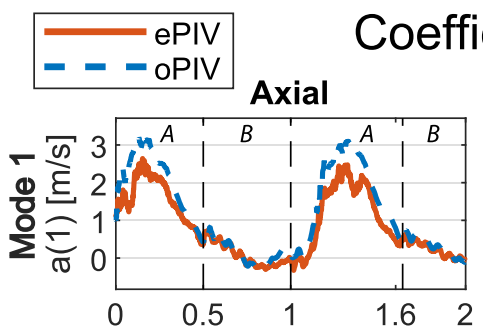

\section{ficients}
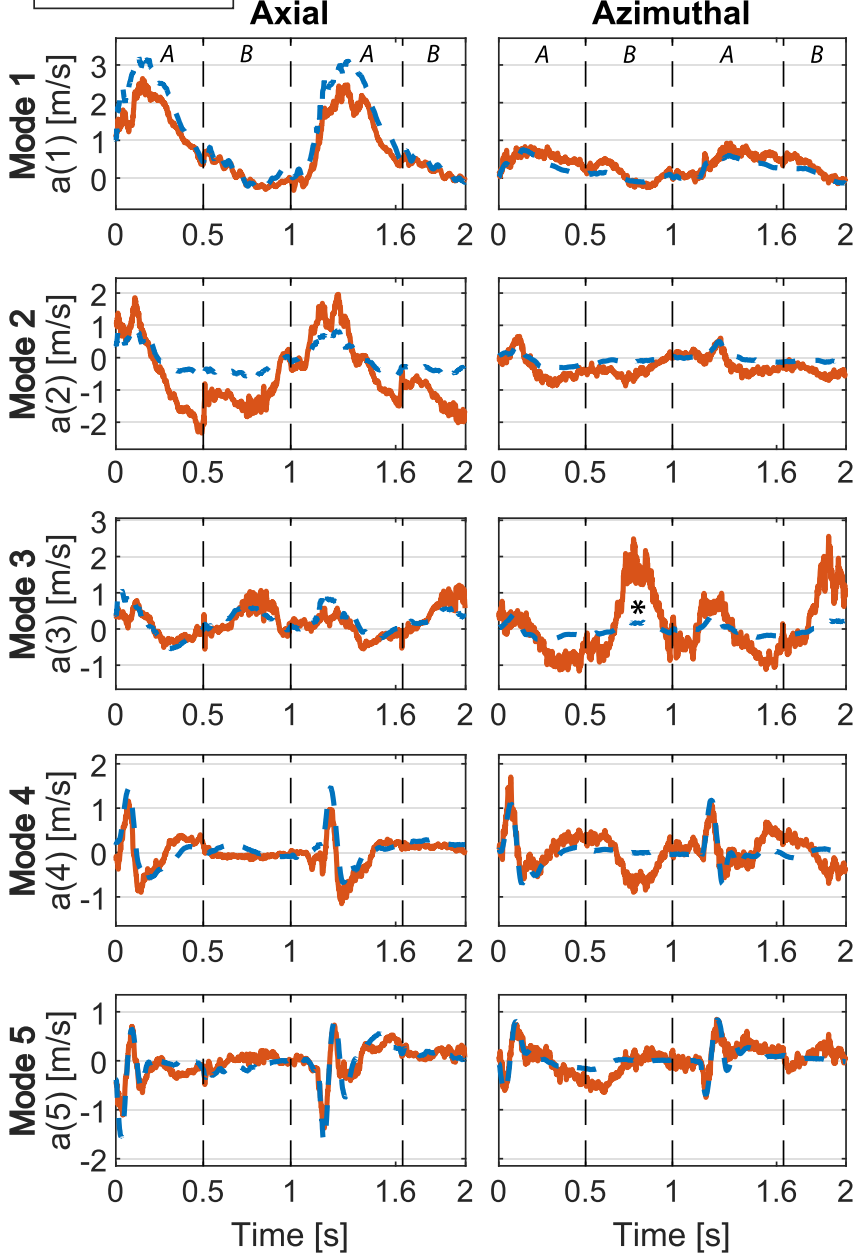

Fig. 6. First five POD coefficients for oPIV and ePIV in the azimuthal and axial directions. Regions demarcated between vertical dotted lines refer to periods associated with filling (A) and ejection (B). * Large difference corresponds to peak ejection period where oPIV estimate is obscured by joint region [see Figs. 5(c) and 1(b)_*'].

already $72 \%$ of the energy within the data set and have an average similarity index of $73 \%$. The first mode's similarity index is $86 \%$. A sharp dip in the similarity index is seen for mode 3 , which corresponds to a mode primarily associated with ejection [Fig. 5(c) and (h)].

Fig. 7(b) shows a similar comparison to Fig. 7(a), except the POD decomposition was performed during frames 1-500 (filling) and 501-1000 (ejection) separately. Note the stronger similarity in filling than ejection, where there is disagreement about the ejection dynamics.

\section{Discussion}

\section{A. Transmitral Jet}

Complex flow patterns, similar to those observed in LVs in vivo, have been measured in an in vitro experimental setup and a high degree of similarity was demonstrated between ePIV and the industry gold standard oPIV. Most notably, 
Rp vs Cum. Energy Fraction: Modes 1-25

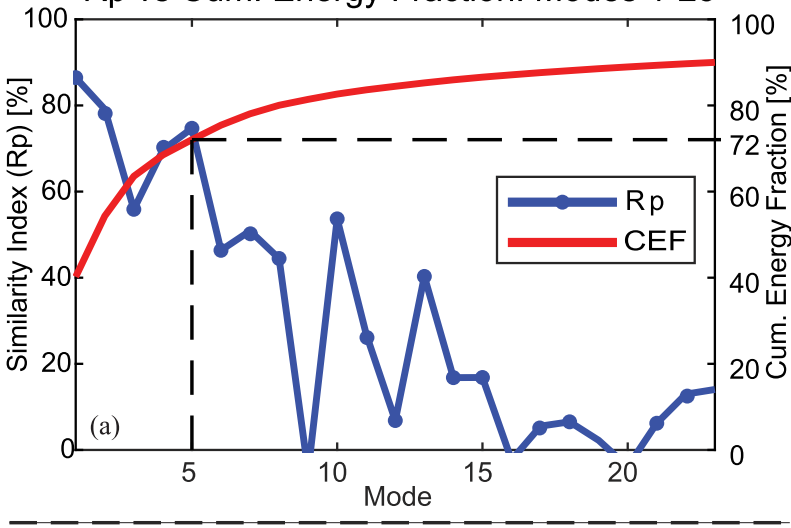

Rp: Filling vs Ejection

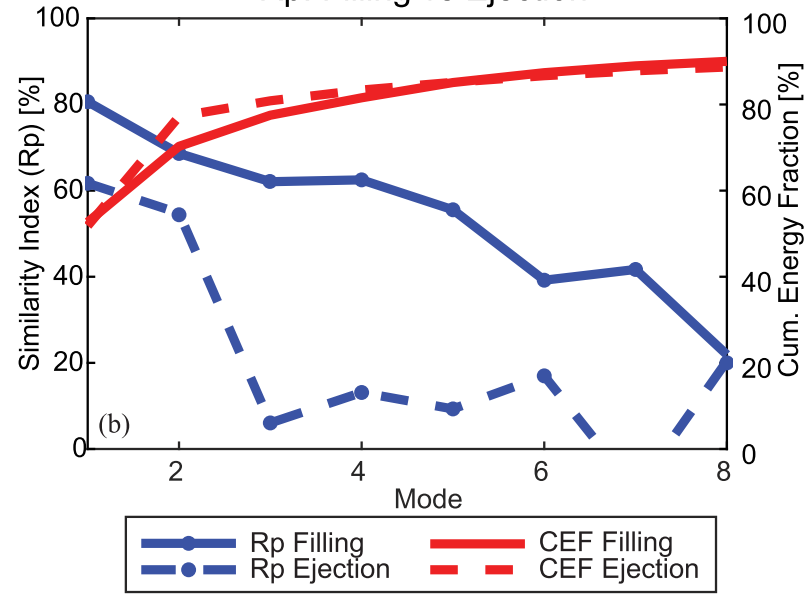

Fig. 7. (a) Rp similarity index versus CEF per mode. $90 \%$ of the energy is contained in the first 25 modes from a data set of 2000 snapshots. Rp is strong for the first five modes, making up $72 \%$ of the combined energy, but drops thereafter. (b) POD decompositions of filling (solid) and ejection (dashed) phases separately. Note the superior similarity in filling versus ejection. This is attributed to the unresolved velocity in the outflow track for the oPIV measurements.

the use of HFR US imaging has enabled ePIV to resolve high velocity flows of approximately $1 \mathrm{~m} / \mathrm{s}$; which was previously not possible with conventional scanning US acquisitions. Figs. 3 and 4 demonstrate that ePIV is able to resolve the high velocity transmitral jet. In similar LV phantom studies, where conventional scanning US-based ePIV was used, the maximum detectable velocities were no higher than $0.45 \mathrm{~m} / \mathrm{s}$ [18], [19].

Although the flow profiles obtained by oPIV and ePIV were similar, ePIV still slightly underestimated the transmitral jet velocity when compared to oPIV. The underestimation of ePIV is likely due to the order of magnitude reduction in spatial resolution between oPIV and ePIV. The transmitral jet is narrow relative to the lateral resolution of the US image and the ePIV interrogation kernels, causing an averaging effect of flow in the region. Thus the flows in the high velocity, narrow jet region, are averaged with the adjacent low velocity flow regions. The ePIV results were also noisier than the oPIV results. The lower SNR of the US images is likely the cause with a SNR of $18 \pm 2 \mathrm{~dB}$ versus $30 \pm 1 \mathrm{~dB}$ for the oPIV images (signal measured inside and noise measured outside the LV). The two tracking errors (Fig. 3(f) and (g)-X) are caused by transient clutter originating from air bubbles on the LV wall. These air bubbles were static but moved in and out of the imaging plane with the motion of the LV wall resulting in stationary but transient speckle patterns which obscured the underlying flow.

\section{B. $P O D$}

POD analysis was able to show a fair agreement between oPIV and ePIV, as can be seen by their similar coefficient vectors for low-order modes (Fig. 6). The slight underestimation of ePIV is also highlighted in the axial coefficients of the first POD mode (Fig. 6-mode 1 and A regions), which constitutes the majority of the transmitral jet energy. However, it can be seen that both ePIV and oPIV follow very similar flow patterns as is attested by the strong similarity indices of their low-order modes.

For mode 2, the flow patterns do show some disparity, where the observed flow moving up the septal (left) wall is wider in structure for ePIV than for oPIV. Also, more flow is present near the outflow tract in the ePIV analysis, which is not present in the oPIV analysis. It may be that, for ePIV, out-of-plane flow is being registered as in-plane flow due to wider US elevational beamwidth in the far-field.

Analyzing separate POD decompositions for the filling and ejection phases show that the two modalities agreed more during filling than ejection. This is due to opaque joint between the outflow tract and the LV, which was a byproduct of the manufacturing process used. The joint obscures the oPIV signal in that region. The loss of similarity with increasing mode number can be explained by a number of factors: first, smaller flow structures are described by higher order modes, where the high resolution of oPIV allows for more detailed flow structures to be captured. Second, the US and laser planes were not perfectly aligned, which would have more impact on higher order modes (due to the smaller flow structures they describe) than lower order modes. Finally, the ePIV estimates were more erratic than the oPIV estimates; this would also affect the higher order modes more than the lower order modes, as the smaller, high-frequency variations would collect in the higher order modes.

\section{Correlation Compounding of Angled Acquisitions}

Recently, HFR ultrasonic imaging has demonstrated value in the study of microvascular flow, where HFRs have been shown to improve Doppler sensitivity to slow flows [34], [35]. This paper uses HFR imaging for the opposite: to track high velocity flows. In cardiac imaging, HFR US is required to maintain speckle pattern coherence between frames, so that the displacement of the microbubble distributions can be tracked. A significant difference in ultrafast processing between the two fields is that angular compounding cannot be performed on the image data, as the speckle displacements are far too large between frames. These large displacements cause loss of coherence when performing spatial (coherent) compounding. In this paper, we instead perform the compounding in the correlation space (Fig. 2), which instead of assuming negligible 


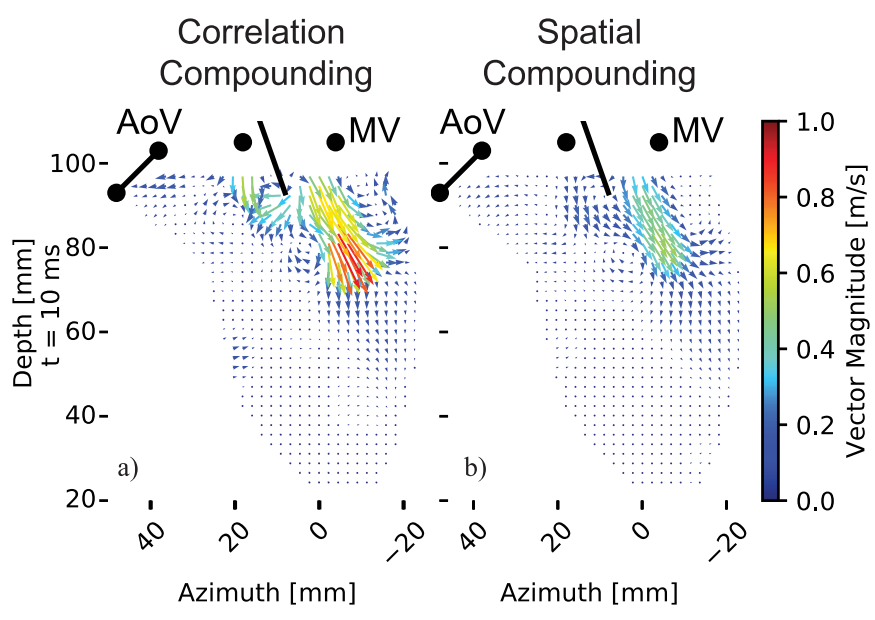

Fig. 8. Effect of compounding angular acquisitions in the (a) correlation domain and (b) spatial domain. When angles are averaged spatially after beamforming (coherent compounding) fast moving scatterers decorrelate, degrading the signal for ePIV analysis. $\mathrm{AoV}=$ aortic valve and $\mathrm{MV}=$ mitral valve.

scatterer displacement between angles; assumes that there is negligible scatterer acceleration between angles. Spatial compounding results in severe underestimation of the transmitral jet, whereas correlation compounding successfully estimates the high velocity dynamics [Fig. 8, see Fig. 3(a) for the oPIV estimate of the same frame]. The superior tracking accuracy of correlation compounding over coherent compounding has also been reported in [36], where it was shown that coherent compounding failed to resolve fast flows where correlation compounding succeeded.

Only three tilting angles were used in this paper, initially as a compromise between speckle decorrelation and sidelobe suppression. With the use of correlation compounding more tilting angles may be viable without affecting speckle decorrelation.

\section{Smoothing}

Limited temporal and spatial smoothing were performed in this paper. This was in an attempt to preserve the high velocity jet flow, which when smoothed, reduced the peak velocities detected. Decreasing the size of the interrogation windows (currently $9 \mathrm{~mm} \times 9 \mathrm{~mm}$ for the finest iteration) would help to reduce smoothing of the peak velocities in the jet; however, we found that halving the interrogation window size $(4.5 \mathrm{~mm} \times 4.5 \mathrm{~mm})$ caused an increase in spurious vector results. The bubble displacement in the jet peaks at about $1.2 \mathrm{~mm} / \mathrm{frame}$, which violates the requirements of the generally accepted "one-quarter rule" for interrogation window size [25]. Alternatively, increasing the overlap percentage (75\% in this paper) would increase the final grid resolution, which would generate a spatially smoother vector field (requiring less smoothing in postprocessing). However, this is accompanied by a considerable computational cost. More advanced velocity field regularization algorithms may prove beneficial in preserving the high velocity flows while removing noisy fluctuations in the velocity field. Gao et al. [19] used a Navier-Stokes-based regularization scheme; however, this method was sensitive to boundary conditions.

\section{E. Limitations}

Whereas great care was taken to align the US and laser planes, the exact orientation of the US plane could not be discerned by eye. These misalignments may mean that flow in one modality is slightly out of plane in the other. This can be alleviated by imaging in 3-D, using matrix transducers for 3-D ePIV, which can be validated against time resolved stereoscopic PIV. A 3-D imaging will also eliminate another limitation of this paper which is that the velocities measured are affected by out-of-plane motion, where scatterers leaving the scanning plane will cause decorrelation between frames, degrading the velocity estimates.

This paper assesses ePIV in an idealized in vitro circumstance. In vivo cardiac imaging will bring more challenges as image quality is further reduced due to attenuation and clutter from the ribs and lungs [37]. Furthermore, the large aperture, curvilinear probe used in this paper would be impractical for transthoracic imaging. The use of a phased array probe with small aperture will bring its own challenges, such as lower resolution and a changing point-spread function with depth due to scan conversion. These issues can be alleviated by performing the ePIV analysis in the polar domain, but this will be explored in detail in the future studies.

Finally, the mechanical index of the transmission pulse used in this paper was 0.09 (measured at a depth of $35 \mathrm{~mm}$ in $22{ }^{\circ} \mathrm{C}$ water). Like in [38], this did not cause any visible bubble disruption but may require optimization for in vivo imaging, where physiological conditions are known to alter bubble stability [39], [40].

\section{CONCLUSION}

This paper has shown that HFR ePIV can accurately estimate the high velocity transmitral jet, in an in vitro LV setting. We have also shown that the dominant flow patterns observed by ePIV and oPIV are very similar.

\section{ACKNOWLEDGMENT}

The authors would like to thank M. Rutten (Ph.D.) for his valuable advice in phantom design. They would also like to thank G. Springeling, M. Manten, A. Brouwer, and R. Beurskens for their assistance in constructing the phantom, F. Mastik for his advice throughout the study, and C. Schinkel for his assistance during the experiments.

\section{REFERENCES}

[1] A. Kheradvar, R. Assadi, A. Falahatpisheh, and P. P. Sengupta, "Assessment of transmitral vortex formation in patients with diastolic dysfunction," J. Amer. Soc. Echocardiogr., vol. 25, no. 2, pp. 220-227, Feb. 2012.

[2] P. Martínez-Legazpi et al., "Contribution of the diastolic vortex ring to left ventricular filling," J. Amer. College Cardiol., vol. 64, no. 16, pp. 1711-1721, Oct. 2014.

[3] G. Pedrizzetti and F. Domenichini, "Left ventricular fluid mechanics: The long way from theoretical models to clinical applications," Ann. Biomed. Eng., vol. 43, no. 1, pp. 26-40, Jan. 2015.

[4] M. Gharib, E. Rambod, A. Kheradvar, D. J. Sahn, and J. O. Dabiri, "Optimal vortex formation as an index of cardiac health," Proc. Nat. Acad. Sci., vol. 103, no. 16, pp. 6305-6308, Apr. 2006.

[5] P. M. Arvidsson et al., "Vortex ring behavior provides the epigenetic blueprint for the human heart," Sci. Rep., vol. 6, no. 1, p. 22021, Apr. 2016. 
[6] A. Pasipoularides, "Mechanotransduction mechanisms for intraventricular diastolic vortex forces and myocardial deformations: Part 1," J. Cardiovascular Transl. Res., vol. 8, no. 1, pp. 76-87, Feb. 2015.

[7] A. Pasipoularides, "Mechanotransduction mechanisms for intraventricular diastolic vortex forces and myocardial deformations: Part 2," J. Cardiovascular Transl. Res., vol. 8, no. 5, pp. 293-318, Jul. 2015.

[8] C. Chnafa, S. Mendez, and F. Nicoud, "Image-based simulations show important flow fluctuations in a normal left ventricle: What could be the implications?" Ann. Biomed. Eng., vol. 44, no. 11, pp. 3346-3358, Nov. 2016.

[9] P. Tortoli, M. Lenge, D. Righi, G. Ciuti, H. Liebgott, and S. Ricci, "Comparison of carotid artery blood velocity measurements by vector and standard Doppler approaches," Ultrasound Med. Biol., vol. 41, no. 5, pp. 1354-1362, May 2015.

[10] K. L. Hansen, H. Møller-Sørensen, J. Kjaergaard, M. B. Jensen, J. A. Jensen, and M. B. Nielsen, "Aortic valve stenosis increases helical flow and flow complexity: A study of intra-operative cardiac vector flow imaging," Ultrasound Med. Biol., vol. 43, no. 8, pp. 1607-1617, Aug. 2017.

[11] K. L. Hansen et al., "Intraoperative cardiac ultrasound examination using vector flow imaging," Ultrason. Imag., vol. 35, no. 4, pp. 318-332, Oct. 2013

[12] J. A. Jensen, "Directional transverse oscillation vector flow estimation," IEEE Trans. Ultrason., Ferroelect., Freq. Control, vol. 64, no. 8, pp. 1194-1204, Aug. 2017.

[13] D. Garcia et al., "Two-dimensional intraventricular flow mapping by digital processing conventional color-Doppler echocardiography images," IEEE Trans. Med. Imag., vol. 29, no. 10, pp. 1701-1713, Oct. 2010.

[14] T. Uejima et al., "A new echocardiographic method for identifying vortex flow in the left ventricle: Numerical validation," Ultrasound Med. Biol., vol. 36, no. 5, pp. 772-788, May 2010.

[15] J. Van Cauwenberge et al., "Assessing the performance of ultrafast vector flow imaging in the neonatal heart via multiphysics modeling and in vitro experiments," IEEE Trans. Ultrason., Ferroelect., Freq. Control, vol. 63, no. 11, pp. 1772-1785, Nov. 2016.

[16] S. Fadnes, S. A. Nyrnes, H. Torp, and L. Lovstakken, "Shunt flow evaluation in congenital heart disease based on two-dimensional speckle tracking," Ultrasound Med. Biol., vol. 40, no. 10, pp. 2379-2391, Oct. 2014.

[17] S. Fadnes, M. S. Wigen, S. A. Nyrnes, and L. Lovstakken, "In vivo intracardiac vector flow imaging using phased array transducers for pediatric cardiology," IEEE Trans. Ultrason., Ferroelect., Freq. Control, vol. 64, no. 9, pp. 1318-1326, Sep. 2017.

[18] C. Prinz et al., "Can echocardiographic particle image velocimetry correctly detect motion patterns as they occur in blood inside heart chambers? A validation study using moving phantoms," Cardiovascular Ultrasound, vol. 10, no. 1, p. 24, Dec. 2012

[19] H. Gao, N. Bijnens, D. Coisne, M. Lugiez, M. Rutten, and J. D'hooge, "2-D left ventricular flow estimation by combining speckle tracking with Navier-Stokes-based regularization: An in silico, in vitro and in vivo study," Ultrasound Med. Biol., vol. 41, no. 1, pp. 99-113, Jan. 2015.

[20] A. Kheradvar et al., "Echocardiographic particle image velocimetry: A novel technique for quantification of left ventricular blood vorticity pattern," J. Amer. Soc. Echocardiogr., vol. 23, no. 1, pp. 86-94, Jan. 2010.

[21] J. Voorneveld et al., "Native blood speckle vs ultrasound contrast agent for particle image velocimetry with ultrafast ultrasound-In vitro experiments," in Proc. IEEE Int. Ultrason. Symp. (IUS), Sep. 2016, pp. $1-4$.

[22] C. H. Leow, E. Bazigou, R. J. Eckersley, A. C. H. Yu, P. D. Weinberg, and M.-X. Tang, "Flow velocity mapping using contrast enhanced highframe-rate plane wave ultrasound and image tracking: Methods and initial in vitro and in vivo evaluation," Ultrasound Med. Biol., vol. 41, no. 11, pp. 2913-2925, Nov. 2015.

[23] C. T. Metz et al., "Regression-based cardiac motion prediction from single-phase CTA," IEEE Trans. Med. Imag., vol. 31, no. 6, pp. 1311-1325, Jun. 2012.

[24] H. A. Kirişli et al., "Evaluation of a multi-atlas based method for segmentation of cardiac CTA data: A large-scale, multicenter, and multivendor study," Med. Phys., vol. 37, no. 12, pp. 6279-6291, Nov. 2010.

[25] W. Thielicke and E. J. Stamhuis, "PIVlab-Towards user-friendly, affordable and accurate digital particle image velocimetry in MATLAB," J. Open Res. Softw., vol. 2, Oct. 2014.

[26] J. Westerweel, "Digital particle image velocimetry-Theory and application," Ph.D. dissertation, Faculty Mech. Maritime Mater. Eng., Delft Univ. Technol., Delft, The Netherlands, 1993.
[27] J. Westerweel and F. Scarano, "Universal outlier detection for PIV data," Exp. Fluids, vol. 39, no. 6, pp. 1096-1100, Dec. 2005.

[28] S. Kefayati and T. L. Poepping, "Transitional flow analysis in the carotid artery bifurcation by proper orthogonal decomposition and particle image velocimetry," Med. Eng. Phys., vol. 35, no. 7, pp. 898-909, Jul. 2013.

[29] K. E. Meyer, J. M. Pedersen, and O. ÖzcaN, "A turbulent jet in crossflow analysed with proper orthogonal decomposition," J. Fluid Mech., vol. 583, p. 199, Jul. 2007.

[30] L. Grinberg, A. Yakhot, and G. E. Karniadakis, "Analyzing transient turbulence in a stenosed carotid artery by proper orthogonal decomposition," Ann. Biomed. Eng., vol. 37, no. 11, pp. 2200-2217, Nov. 2009.

[31] A. Chatterjee, "An introduction to the proper orthogonal decomposition," Current Sci., vol. 78, no. 7, pp. 808-817, 2000

[32] K. E. Meyer, D. Cavar, and J. M. Pedersen, "POD as tool for comparison of PIV and LES data," in Proc. 7th Int. Symp. Particle Image Velocimetry, 2007, pp. 1-12.

[33] L. Sirovich, "Turbulence and the dynamics of coherent structures part I: Coherent structures," Q. Appl. Math., vol. XLV, no. 3, pp. 561-571, 1987.

[34] C. Demené et al., "Spatiotemporal clutter filtering of ultrafast ultrasound data highly increases Doppler and ultrasound sensitivity," IEEE Trans. Med. Imag., vol. 34, no. 11, pp. 2271-2285, Nov. 2015.

[35] B.-F. Osmanski, S. Pezet, A. Ricobaraza, Z. Lenkei, and M. Tanter, "Functional ultrasound imaging of intrinsic connectivity in the living rat brain with high spatiotemporal resolution," Nature Commun., vol. 5, p. 5023 , Oct. 2014

[36] C. H. Leow and M.-X. Tang, "Spatio-temporal flow and wall shear stress mapping based on incoherent ensemble-correlation of ultrafast contrast enhanced ultrasound images," Ultrasound Med. Biol., vol. 44, no. 1, pp. 134-152, Jan. 2018.

[37] A. Fatemi, H. Torp, S. Aakhus, and A. Rodriguez-Molares, "Increased clutter level in echocardiography due to specular reflection," Proc. SPIE, Med. Imag., Ultrason. Imag. Tomogr., vol. 10139, p. 101391D, Mar. 2017.

[38] M. Toulemonde, R. J. Eckersley, and M.-X. Tang, "High frame rate contrast enhanced echocardiography: Microbubbles stability and contrast evaluation," in Proc. IEEE Int. Ultrason. Symp. (IUS), Sep. 2017, pp. $1-4$.

[39] M. Itani and R. F. Mattrey, "The effect of inhaled gases on ultrasound contrast agent longevity in vivo," Mol. Imag. Biol., vol. 14, no. 1, pp. 40-46, Feb. 2012.

[40] H. Mulvana, E. Stride, J. V. Hajnal, and R. J. Eckersley, "Temperature dependent behavior of ultrasound contrast agents," Ultrasound Med. Biol., vol. 36, no. 6, pp. 925-934, Jun. 2010.

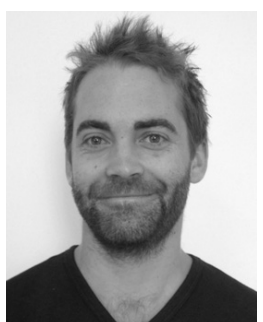

Jason Voorneveld (GS'16) was born in Johannesburg, South Africa, in 1987. He received the B.Sc. degree in electro-mechanical engineering and the M.Sc. degree in biomedical engineering from the University of Cape Town, Cape Town, South Africa, in 2009 and 2014, respectively. He is currently pursuing the $\mathrm{Ph} . \mathrm{D}$. degree in biomedical engineering with Erasmus MC, Rotterdam, The Netherlands.

His research interests include high frame rate ultrasound imaging, blood flow imaging, and ultrasound image/signal processing.

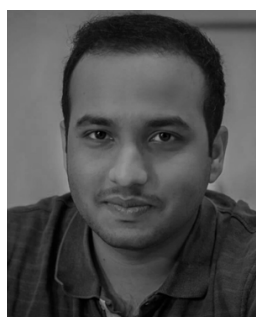

Aswin Muralidharan was born in Calicut, India, in 1993. He received the B.Tech. degree in chemical engineering from the National Institute of Technology, Calicut, in 2014, and the M.Sc. degree in chemical engineering specializing in transport phenomena from the Delft University of Technology, Delft, The Netherlands, in 2017, where he is currently pursuing the Ph.D. degree with the Product and Process Engineering Research Group, Department of Chemical Engineering.

His research interests include biophysics, soft condensed matter physics, and transport phenomena. 


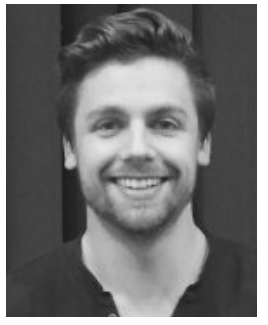

Timothy Hope was born in Johannesburg, South Africa, in 1987. He received the B.Sc. degree in electro-mechanical engineering and the M.Sc. degree in mechanical engineering from the University of Cape Town, Cape Town, South Africa, in 2009 and 2015, respectively.

He worked in east Africa and Brazil as a wireline field engineer for Schlumberger, an oilfield service provider, from 2010 to 2012 . He is currently the Project Manager of research and development with Mamapey Offshore Industries, Dordrecht, The Netherlands. His research interests include developing automated mooring solutions for the maritime industry.

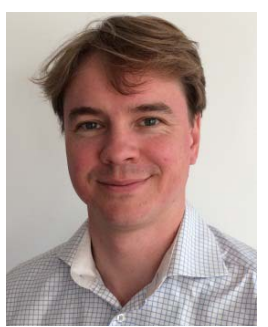

Hendrik J. Vos (M'14) received the M.Sc. degree in applied physics from Delft University of Technology, Delft, The Netherlands, in 2004, and the Ph.D. degree from the Department of Biomedical Engineering, Erasmus MC, Rotterdam, The Netherlands, in 2010 .

He was a Postmaster Researcher with the University of Florence, Florence, Italy, and a Contract Researcher for the petrochemical industry, focussing on cutting-edge ultrasonic solutions. He is currently an Assistant Professor with Erasmus MC. His research interests include acoustical array technology for biomedical imaging in all its aspects: transducers, 2-D and 3-D beamforming, cardiac shear waves, ultrafast Doppler, contrast imaging, and related subclinical and clinical studies.

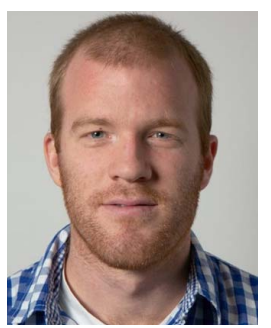

Pieter Kruizinga (M'16) received the B.Sc. degree in biomedical engineering from the University of Twente, Enschede, The Netherlands, in 2006, the M.Sc. degree in biomedical engineering from Delft University of Technology, Delft, The Netherlands, in 2010, and the Ph.D. degree from the Department of Biomedical Engineering of the Thorax Center, Erasmus MC, Rotterdam, The Netherlands, in 2015.

He was a Researcher with the Ultrasound Imaging and Therapeutics Research Laboratory, University of Texas at Austin, Austin, TX, USA. His current research interests include high frame rate imaging in 3-D, compressive sensing, and functional ultrasound.

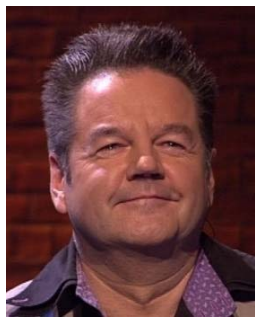

Antonius F. W. van der Steen (M'94-SM'03-F'13) received the M.Sc. degree in applied physics from Delft University of Technology, Delft, The Netherlands, in 1989, and the Ph.D. degree in medical sciences from the University of Nijmegen, Nijmegen, The Netherlands, in 1994.

Since 1994, he has been with the Thorax Centre, Erasmus MC, Rotterdam, The Netherlands, and the Interuniversity Cardiology Institute of the Netherlands, Utrecht, The Netherlands. Since 2002, he has been a Full Professor and the Head of biomedical engineering with Erasmus MC. Since 2013, he has been a Full Professor of applied physics with the Delft University of Technology. Since 2013, he has also been an Honorary Visiting Professor with the Shenzhen Institute of Advanced Technologies, Chinese Academy of Sciences, Shenzhen, China. $\mathrm{He}$ is co-founder of the Medical Delta, a collaboration between Erasmus MC, TU Delft, and Leiden University Medical Centre. His research interests include diagnostic cardiologic imaging devices with emphasis on echography, image-guided catheter-based treatment of atherosclerosis, ultrasound contrast agents, ultrasound transducers, and vascular biomechanics.

$\mathrm{He}$ is a member of the Netherlands Academy of Engineering and a member of the Netherlands Academy of Sciences. He is a fellow of the European Society of Cardiology. He was a recipient of the 2000 NWO PIONIER Technical Sciences (Best Researcher of The Netherlands in technical science under 40) and the 2007 Simon Stevin Master (Best Researcher in translational research). He was the IEEE UFFC Distinguished Lecturer from 2011 to 2012. From 2014 to 2017, he was the Chairman of the board of the Dutch Technology Foundation and the NWO technical sciences.

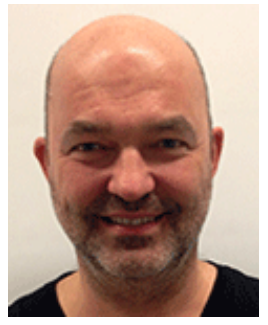

Frank J. H. Gijsen received the Ph.D. degree from Eindhoven University of Technology, Eindhoven, The Netherlands, with a focus on the modeling of blood flow in large arteries.

He was a founder of a bioengineering company, and he was involved as a board member in setting up the Department of Biomedical Engineering, Eindhoven University of Technology, Eindhoven, The Netherlands. He joined Erasmus MC, Rotterdam, The Netherlands, in 2001, as a Post-Doctoral Researcher and is currently Associate Professor with the Department of Biomedical Engineering, Thorax Center, Erasmus MC. His research interests include image-based biomechanics of the cardiovascular system, influence of blood flow-induced wall shear stress on plaque progression, composition, stability, and rupture.

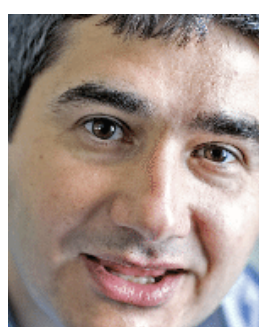

Sasa Kenjeres was born in Kakanj, Bosnia and Herzegovina, in 1967. He received his Ph.D. degree in applied physics from Delft University of Technology, Delft, The Netherlands, in 1999.

From 1992 to 1995 , he was a Visiting Scientist in fluid mechanics at the Friedrich-Alexander University Erlangen-Nuremberg, Germany. From 2001 to 2006, he was a Research Fellow with the Royal Netherlands Academy of Sciences and Arts, Amsterdam, The Netherlands. From 2006 to 2012, he was an Assistant Professor at the Faculty of Applied Sciences, Department of Multi-Scale Physics, Delft University of Technology. During 2005-2006, he was a Burgers Visiting Associate Professor at the University of Maryland, College park, MD, USA. During 2007-2013, he was a Marie-Curie Visiting Professor at the AGH University of Science and Technology, Krakow, Poland. During 2002-2008, he was a Visitor with the ERCOFTAC, Zürich, Switzerland, and Leonhard Euler Center, ETH Zurich, Zürich. Since 2012, he has been an Associate Professor with the, Faculty of Applied Sciences, Department of Chemical Engineering, Transport Phenomena Section, Delft University of Technology. His research interests include modeling, computer simulations and experiments of advanced fluid dynamics, heat and mass transfer, and turbulence.

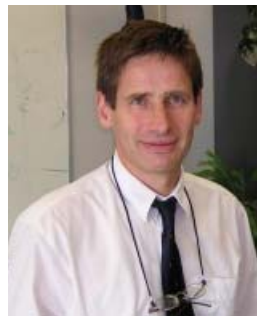

Nico de Jong (A'97-M'09) received the M.Sc. degree in physics with a specialization in pattern recognition from Delft University of Technology, Delft, The Netherlands, in 1978, and the Ph.D. degree with a specialization in acoustic properties of ultrasound contrast agents from the Department of Biomedical Engineering, Thorax Center, Erasmus Medical Center (Erasmus MC), The Netherlands, in 1993.

Since 1980, he has been a Staff Member with the Thorax Center, Erasmus MC. Since 2011, he has been a Professor of molecular ultrasonic imaging and therapy with Erasmus MC and Delft University of Technology. Since 2015, he has been the part-time Head of the Department of Acoustical Waveform Imaging, Delft University of Technology.

Dr. de Jong is the Organizer of the Annual European Symposium on Ultrasound Contrast Imaging, Rotterdam. He is on the Safety Committee of the World Federation of Ultrasound in Medicine and Biology, and is an Associate Editor of the Ultrasound in Medicine and Biology and a Guest Editor of special issues of different journals.

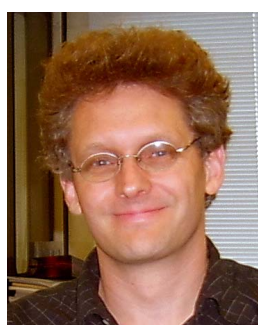

Johan G. Bosch (M'07) received the M.Sc. degree in electrical engineering from Eindhoven University of Technology, Eindhoven, The Netherlands, in 1985, and the Ph.D. degree from Leiden University Medical Center, Leiden, The Netherlands, in 2006.

$\mathrm{He}$ is currently an Associate Professor and staff member with the Department of Biomedical Engineering, Thoraxcenter, Erasmus MC, Rotterdam. He is specialized in 2-D and 3-D echocardiographic image processing/analysis and transducer development. His main research interests are optimal border detection approaches, geometrical and statistical models, and anatomical and physical knowledge representations for border detection. 